\section{Assessment of a novel pinhole supplementary implant for sulcus fixation in pseudophakic cadaver eyes}

KT Tsaousis ${ }^{1}$, L Werner ${ }^{1}$, CLC Trindade ${ }^{2}$, J Guan ${ }^{1}$, J Li ${ }^{1}$ and N Reiter ${ }^{1}$
Purpose To evaluate a novel small-aperture supplementary implant that applies the principle of pinholes for management of irregular astigmatism, in postmortem human eyes.

Methods Pseudophakic human cadaver eyes were imaged by anterior segment optical coherence tomography (AS-OCT) to assess position of the in-the-bag intraocular lens (IOL). Eyes were prepared as per the MiyakeApple technique. Two versions of the supplementary implant (open-loop and tripod designs) were then inserted into the sulcus of each eye. Evaluations under AS-OCT and from anterior and posterior views of the anterior segment were used to assess IOL fixation, centration, tilt, and interlenticular distance (ILD). This experimental study has been conducted in John A. Moran Eye Center, University of Utah.

Results Nine eyes were selected, with various sizes, primary IOL materials/designs, and Soemmering's ring formation. The openloop model exhibited a mild degree of decentration and tilt in 2 eyes with zonular dehiscence. Mild decentration and tilt of the tripod were observed in 4 eyes; in 1 additional eye it was centered but mildly tilted. Three eyes with zonular dehiscence had one of the closed loops of the tripod located posteriorly to the ciliary processes. In all cases, an ILD was observed between the lenses (open loop: $0.65 \pm 0.13 \mathrm{~mm}$; tripod: $0.41 \pm 0.12 \mathrm{~mm}$ ).

Conclusions It is important to take into account anatomical aspects related to ciliary sulcus fixation of supplementary IOLs. Both designs evaluated, exhibited appropriate centration and ILD. The open-loop design had less risk of tilt in association with haptics protruding posteriorly through areas of zonular weakness.

Eye (2018) 32, 637-645; doi:10.1038/eye.2017.239; published online 27 October 2017

Introduction

Best-corrected visual acuity after penetrating keratoplasty is frequently limited by the occurrence of irregular corneal astigmatism, a condition that affects $\sim 20 \%$ of cases. ${ }^{1,2}$ This is not tolerated by the majority of patients, whose visual ability is severely compromised. ${ }^{3}$ Several methods have been proposed for the management of residual post keratoplasty irregular astigmatism, such as laser in situ keratomileusis (LASIK) and arcuate keratotomy, ${ }^{4,5}$ photorefractive keratectomy, ${ }^{6}$ as well as femtosecond laser-assisted astigmatic keratotomy. ${ }^{7}$ Evidently, in many instances laser is not available or appropriate and use of rigid contact lenses is the ultimate solution. ${ }^{8}$ The most common problems regarding contact lens use are related to intolerance and increased risk of infection. A novel approach to this problem is the advent of a small-aperture supplementary implant, which applies the pinhole principle for improvement of visual acuity and dysphotopsia symptoms, such as glare. The pinhole principle is based on the barring of peripheral light rays to prevent them from reaching the retina.

Consequently, the result is improved image resolution since central (paraxial) light rays are not significantly affected by optical system's aberrations. The principle of small-aperture correction is commonly applied in ophthalmology for diagnostic purposes but has also been commercially applied throughout cornea inlays for presbyopia management. ${ }^{9,10}$
${ }^{1}$ Department of Ophthalmology and Visual Sciences, John A. Moran Eye Center, University of Utah, Salt Lake City, UT, USA

${ }^{2}$ Cançado-Trindade Eye Institute, Belo Horizonte, Brazil

Correspondence:

L Werner, John A. Moran Eye Center, University of Utah, 65 Mario Capecchi Drive, Salt Lake City, UT 84132, USA

Tel: +1 8015816586 ; Fax: +1 8015813357 E-mail: liliana.werner@hsc. utah.edu

Received: 17 March 2017 Accepted in revised form: 17 July 2017 Published online: 27 October 2017 
Trindade and Trindade have recently published a case report about the use of this novel pinhole implant in a patient with Urrets-Zavalia syndrome (diffuse iris atrophy after penetrating keratoplasty). ${ }^{11}$ Clinical results were very encouraging, and consequently the use of the small-aperture supplementary implant is expected to widen since additional indications could include any combined ametropia (residual spherical and cylindrical error) in a pseudophakic eye after penetrating keratoplasty.

The small-aperture supplementary implant was designed to be fixated in the ciliary sulcus of pseudophakic eyes. While different reports assessed the visual function of the patients after implantation of supplementary intraocular lenses (IOLs), ${ }^{12,13}$ it is important to take into account anatomical aspects related to ciliary sulcus fixation of these lenses. ${ }^{14,15}$ Therefore, the aim of this study was to assess the new supplementary implant in human cadaver eyes. Our chief objective was the investigation of parameters vital for its clinical performance such as fixation, centration, tilt and estimation of interlenticular distance (ILD - clearance in relation to the primary, in-the-bag IOL).

\section{Materials and methods}

The pinhole implant (XtraFocus, Morcher GmbH, Germany) has the overall design of an IOL, but without any refractive power. Because the design of the pinhole implant has progressively evolved since its first description, two versions were evaluated in this study: 93L (newer open-loop model), and 93E (older tripod
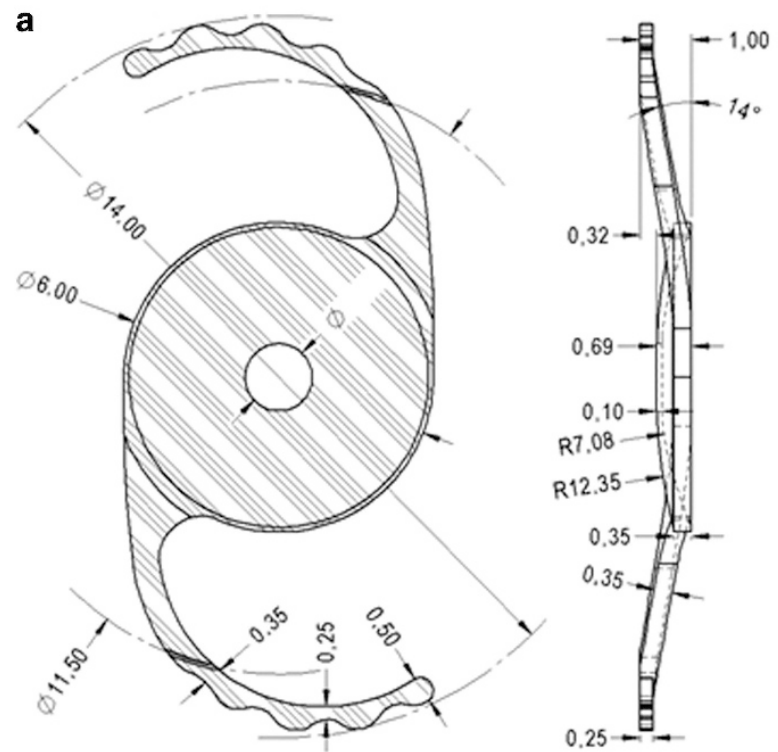

model). Figure 1 shows overall characteristics of both models. Both lenses were made of a black hydrophobic acrylic material, had a concave-convex design, as well as a posterior optic-haptic angulation. The newer model (93L) was an open-loop design, had an overall diameter of $14.0 \mathrm{~mm}$, a $6.0 \mathrm{~mm}$ occlusive portion with a $1.3 \mathrm{~mm}$ central opening, an optic-haptic angulation of 14 degrees, and a thinner occlusive portion. The older model (93E) was a tripod design with 3 closed loops, had an overall diameter of $13.5 \mathrm{~mm}$, a $6.0 \mathrm{~mm}$ occlusive portion with a $1.5 \mathrm{~mm}$ central opening, and an optic-haptic angulation of 14 degrees.

This study was performed in collaboration with eye banks nationwide within the US, and the methodology was similar to previous studies in our laboratory evaluating other supplementary IOLs. ${ }^{14,15}$ Pseudophakic human eyes obtained postmortem, were immersed in $10 \%$ neutral buffered formalin upon enucleation. Interval between death of the donor and enucleation was no longer than $24 \mathrm{~h}$. Gross measurements were obtained through a digital metric ruler (model Absolute Digimatic, Mitutoyo Corp., Aurora, IL, USA), including anteriorposterior length, equatorial diameter, and corneal diameter. The eyes were then bisected coronally just anterior to the equator. Gross examination and photographs of the anterior segment were done from the posterior aspect (Miyake-Apple view) to assess the type and fixation of the IOL implanted, as well as the severity of Soemmering's ring formation, and status of the zonular apparatus. $^{16}$

Eyes with in-the-bag fixated IOLs were selected for inclusion in the study. The anterior segment of each

b

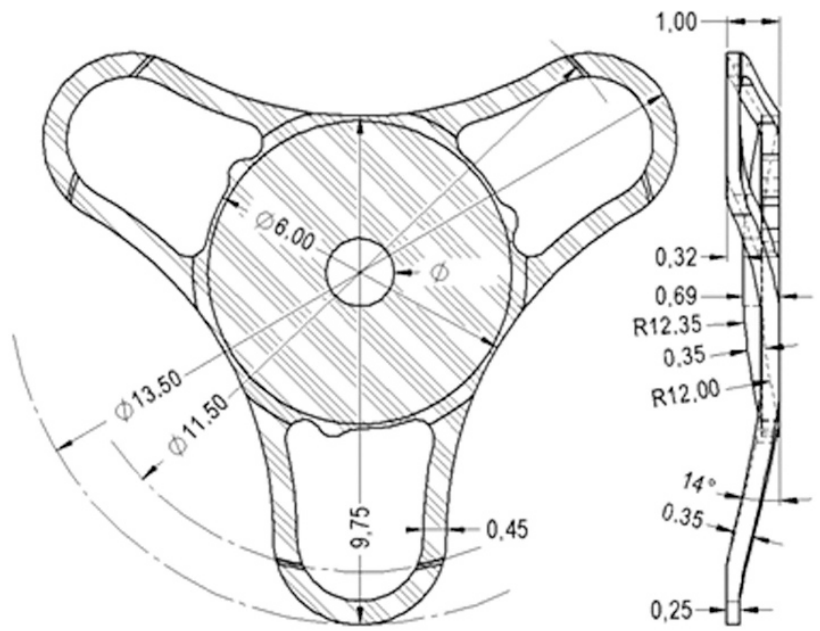

Figure 1 Schematic drawings showing the overall design of the implants evaluated in this study. (a) Model 93L (newer open-loop model). (b) Model 93E (older tripod model). Provided by Morcher GmbH. 


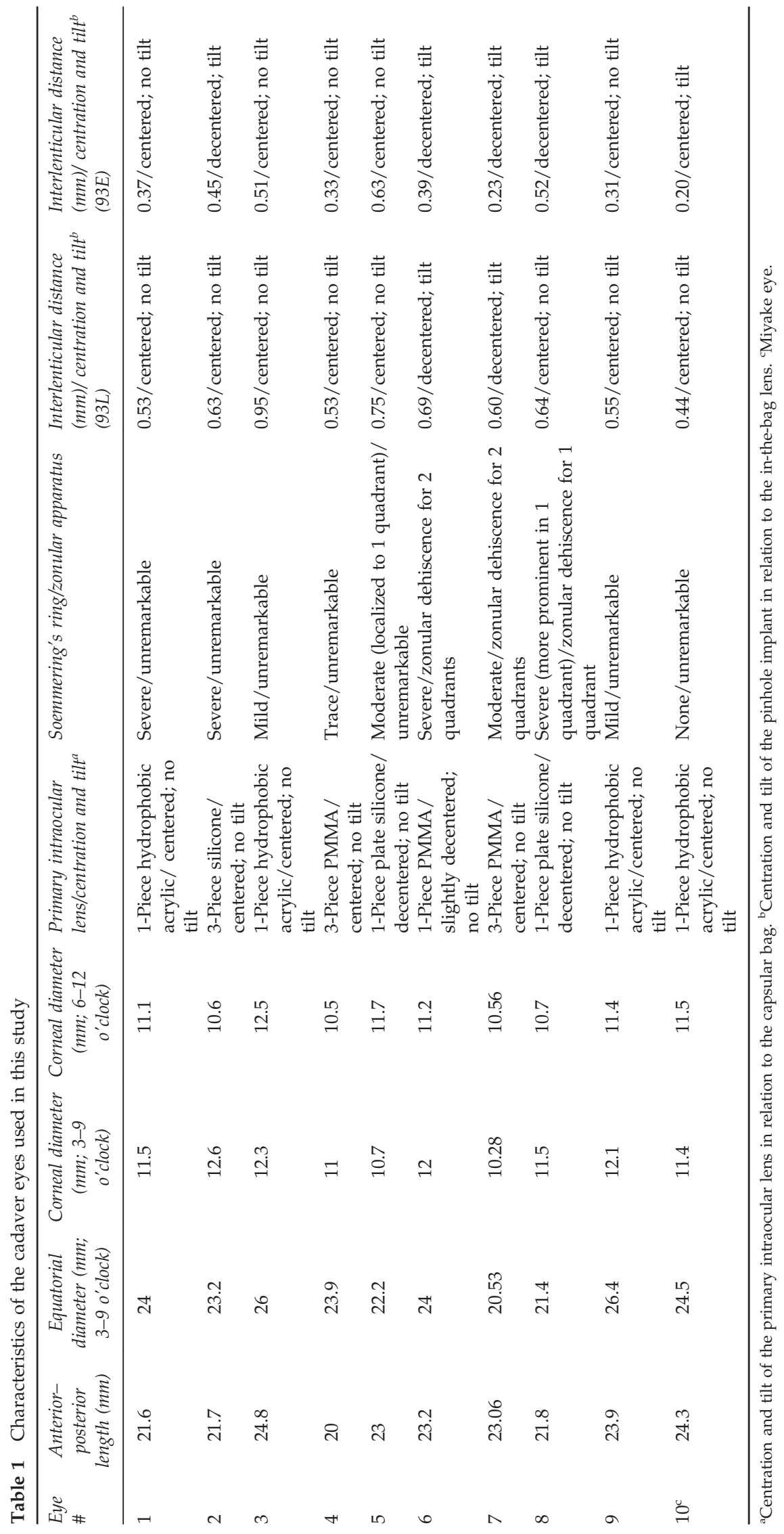


selected eye was then glued to a glass slide according to the Miyake-Apple technique, and underwent preoperative examination under the Visante anterior segment optical coherence tomography (AS-OCT; Carl Zeiss Meditec, Jena, Germany) to assess centration and tilt of the primary IOL. The cornea was then removed with scissors. The iris was also partially removed for 360 degrees with scissors; a peripheral rim of iris was kept to simulate a pupil dilation of $\sim 8.0 \mathrm{~mm}$. Ophthalmic viscosurgical device (OVD) was injected behind the iris. The add-on implant was then inserted behind the iris with a forceps and positioned in the sulcus. Residual OVD was then removed by irrigation and aspiration (Infinity, Alcon, Fort Worth, TX, USA). In each eye, one of the models was inserted first, analyzed, and then explanted, with subsequent insertion of the other model.

The eyes underwent post-implantation evaluation with the Visante AS-OCT, with measurement (in $\mathrm{mm}$ ) of the ILD between the study implant and the primary IOL. The remaining peripheral iris was then completely removed and the position of each haptic element was analyzed under the operating microscope from anterior and posterior views of the anterior segment. Photographs were obtained for documentation. The analyses focused on the fixation, centration, and tilt of the add-on implant, as well as on the interlenticular space between the primary in-the-bag IOL and the add-on.

A fresh, phakic human eye obtained postmortem within $72 \mathrm{~h}$ of enucleation from a 65-year-old male donor was also prepared according to the Miyake-Apple technique for experimental surgery. ${ }^{16}$ After phacoemulsification, and irrigation/aspiration, a singlepiece hydrophobic acrylic IOL (AcrySof, Alcon, Fort Worth, TX, USA) was implanted within the capsular bag. Gross analyses of this eye from the anterior and posterior views, as well as analyses under the AS-OCT were performed before and after insertion of each model of the add-on implant. Inclusion of this eye allowed analyses of both versions of the implant in fresh, non-fixated tissue. Also, the completely clean capsular bag without any Soemmering's ring formation provided a better evaluation of the pinhole implants from the posterior view of the anterior segment.

ILD results were expressed as mean \pm SD. The F-test two-sample for variances was first used to confirm that variances in both groups of implant versions were similar. A $t$-test paired two sample for means was then used to assess significant differences between both study implant versions in terms of ILD. A $P$ value $<0.05$ was considered statistically significant.

\section{Results}

Nine pseudophakic eyes were selected for inclusion in this study based on gross measurements, the type of primary in-the-bag implanted IOL, as well as the degree of Soemmering's ring formation. Eyes were chosen so as to have a wide variety of sizes, primary IOL materials and designs, and degrees of Soemmering's ring formation in order to replicate the many possible clinical scenarios that the study add-on lenses might encounter. A total of 20 eyes obtained postmortem and fixated in formalin upon enucleation were evaluated as part of the inclusion process. After evaluating 20 eyes, the 9 eyes selected (Table 1) appeared to provide such variety, as described above. Some of the eyes were not included due to sulcus fixation of an IOL, or asymmetric fixation (bag/sulcus).

Table 1 shows the overall characteristics of the 9 formalin-fixated, pseudophakic eyes included. The mean age of the donors was $83 \pm 11$ years. The mean anteriorposterior length, horizontal equatorial diameter, and horizontal corneal diameter of the eyes were $22.56 \pm 1.43$, $23.51 \pm 1.94$, and $11.55 \pm 0.77 \mathrm{~mm}$, respectively. The following primary IOLs were represented: 1-piece hydrophobic acrylic $(N=3), 1$-piece plate silicone $(N=2)$, 3-piece silicone $(N=1), 3$-piece PMMA $(N=2)$, and 1piece PMMA $(N=1)$. Soemmering's ring formation was as follows: trace $(N=1)$, mild $(N=2)$, moderate $(N=2)$ and severe $(N=4)$. None of the in-the-bag lenses had any tilt, but three of them exhibited decentration. Two eyes had Soemmering's ring formation localized or more prominent in 1 quadrant. Three eyes exhibited localized areas of zonular dehiscence under gross evaluation. Figure 2 shows Miyake-Apple views of some of the formalin-fixated, pseudophakic eyes included in this study.

In eyes \#1 to \#5, the new model was placed first, removed, and then the old model was placed. In eyes \#6 to \#9, the old model was placed first, and then the new model. The new model exhibited a mild degree of decentration and tilt (in relation to the primary in-the-bag IOL) only in two eyes with zonular dehiscence (eyes \#6 and \#7). Mild decentration and tilt of the old design were observed in four eyes; in one additional eye the old design was centered but mildly tilted (Figure 3). Gross evaluation showed the positioning of the haptic elements of both implant models in the sulcus (Figures $4 \mathrm{a}$ and b). The three eyes with zonular dehiscence had one of the closed loop haptics of the old model located posteriorly to the ciliary processes through the area of zonular dehiscence, while the other closed loops were fixated in the sulcus.

In all 9 cases, an interlenticular space was observed between the primary-in-the-bag IOL and the add-on implant. The ILD between the study implant and the 

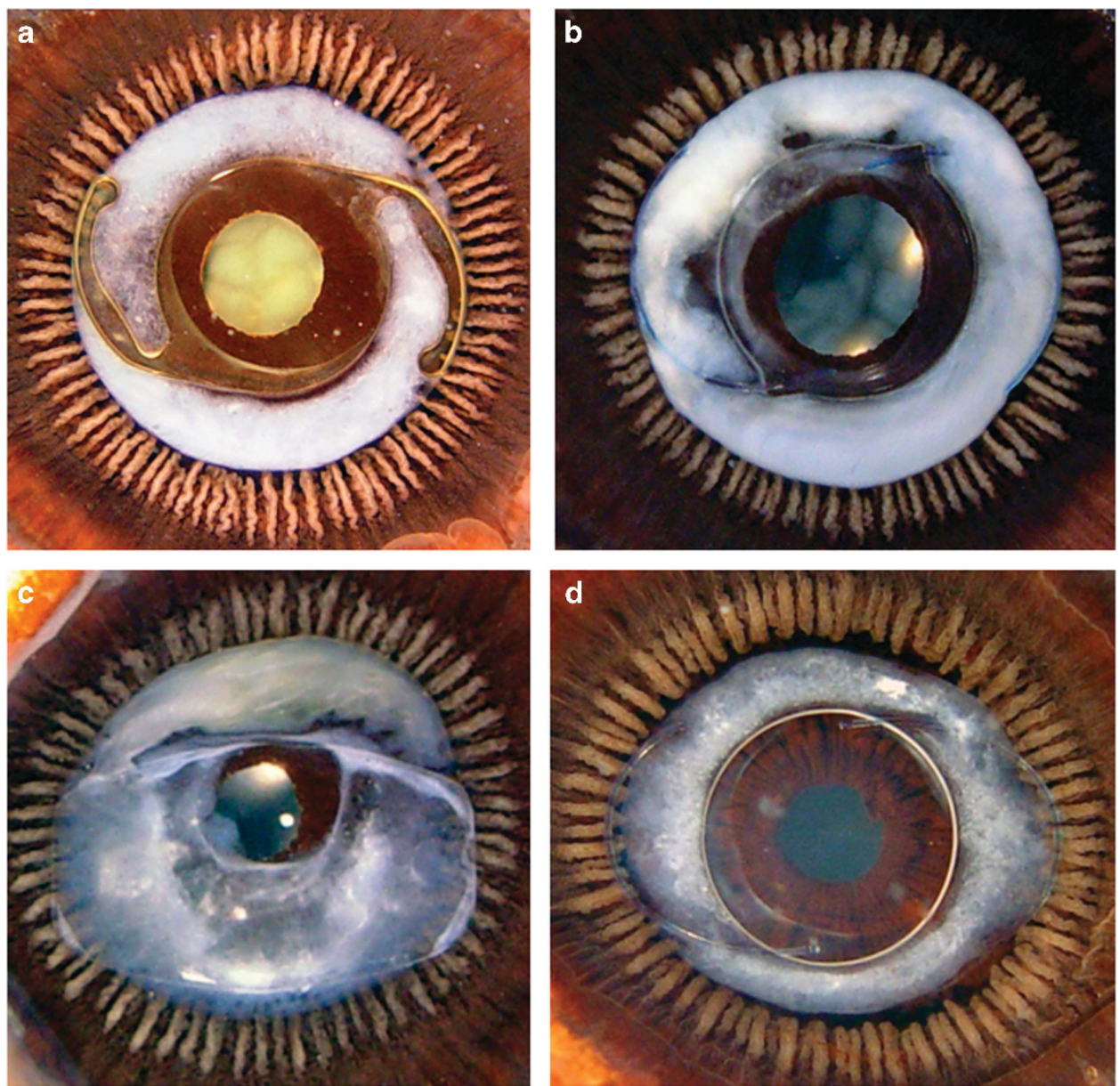

Figure 2 Gross photographs from the Miyake-Apple view of representative formalin-fixated, pseudophakic human eyes obtained postmortem used in this study. (a-d): Eyes \#1, \#2, \#5, and \#7, respectively (Table 1).

primary IOL (measured at the edge of the pinhole) was always greater for the new model in comparison with the old model, likely due to the differences in thickness of the occlusive portion of both models.

Characteristics of the fresh eye used in this study (\#10), are also shown in Table 1. Analyses of this eye showed that the new model was centered and exhibited no tilt (Figures $4 \mathrm{c}$ and d). The old model was centered, and a mild tilt was observed under AS-OCT examination, on account of the presence of one of the closed loops posteriorly to the ciliary processes, through a localized area of zonular dehiscence observed after insertion of this add-on implant (Figures 4e-h).

Considering only the 9 formalin-fixated, pseudophakic eyes evaluated in this study, the mean ILD for the open-loop implant was $0.65 \pm 0.13 \mathrm{~mm}$, and for the tripod was $0.41 \pm$ $0.12 \mathrm{~mm}$ (two-tail $P=0.0002$; paired $T$-test). Considering all 10 eyes evaluated in this study, the mean ILD for the openloop implant was $0.63 \pm 0.14 \mathrm{~mm}$, and for the tripod was $0.39 \pm 0.13 \mathrm{~mm}$ (two-tail $P<0.0001$; paired $T$-test).

\section{Discussion}

Patients that undergo ophthalmic surgeries nowadays have high expectations regarding their final visual ability. This observation applies mainly for cataract operations but also for patients that undergo penetrating keratoplasty. While in phacoemulsification procedures postoperative refractive error is not so common, in penetrating keratoplasty, irregular astigmatism is present in the majority of cases. ${ }^{1-3}$ Given that other options of management, such as ophthalmic laser is not always available or appropriate, implantation of an add-on lens appears to be an attractive alternative. Piggyback implantation is an easier operation rather than an IOL exchange. Especially in eyes with penetrating keratoplasty, it would be difficult to correct all complicated optical aberrations by the means of a new IOL since irregular astigmatism and other induced aberrations would compromise the quality of vision. Trindade and Trindade have reported a favorable result in a patient with irregular corneal astigmatism and severe 

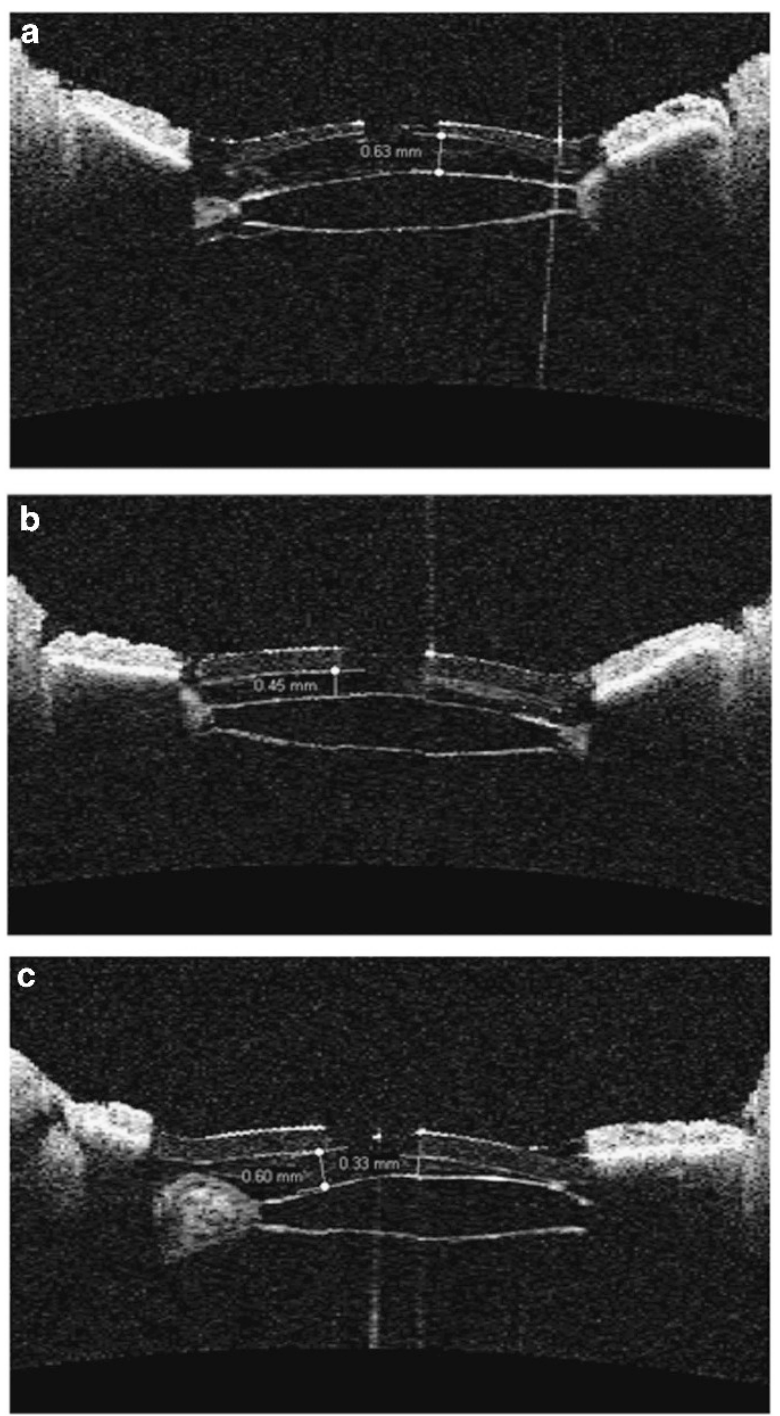

Figure 3 AS-OCT images obtained from eye \#2 after insertion of the add-on implants. Values shown in $\mathrm{mm}$ are ILD measurements. (a) Model 93L is centered and exhibits no tilt in relation to the in-the-bag primary IOL. (b) Model 93E is centered and exhibits no tilt in the horizontal meridian. (c) Analysis of the vertical meridian shows mild decentration and tilt of model 93E in relation to the in-the-bag primary IOL.

light sensitivity after penetrating keratoplasty and UrretsZavalia syndrome. ${ }^{11}$ Corrected distance visual acuity (CDVA) $\left(-3.50-4.25 \times 90^{\circ}\right)$ was $20 / 200$ and the patient used rigid contact lens, which improved his VA to $20 / 50$. After implantation of the pinhole implant, patient's CDVA improved to $20 / 30\left(-3.00-4.25 \times 120^{\circ}\right)$ and the use of contact lens was discontinued resulting in better quality of life overall.

A video recently presented at the Annual Meeting of the American society of Cataract and Refractive Surgery (ASCRS) described results from 24 eyes implanted with the pinhole implant, with a mean follow up of 22 months. ${ }^{17}$ Main indications included cases of irregular astigmatism post radial keratotomy, post penetrating keratoplasty, or associated with keratoconus. Visual field constriction was not spontaneously reported by any of the patients. Visual field analysis revealed a subtle reduction of overall sensitivity (with a magnitude of $\sim 2 \mathrm{~dB}$ ), without clinical relevance. A subjective perception of visual field constriction caused by pinhole occlusion is determined by 2 distinct factors: pinhole diameter, and distance to the eye's nodal point. ${ }^{18}$ In this specific device, the $1.3 \mathrm{~mm}$ aperture is placed very close to the nodal point of the eye, thus minimizing its impact on visual field. ${ }^{17}$ The visual field constriction observed with external pinhole occlusion (eg, pinhole glasses) does not represent the same situation, since with this method the pinhole is placed in a distant plane from the nodal point. Also, a pupil diameter of $\sim 1.5 \mathrm{~mm}$ is not a rare finding in older patients, and they usually do not report any visual field complaints. ${ }^{19}$ Therefore, we believe that the size of the aperture is not small enough (and not distant enough to the nodal point) to represent a clinical threat to visual field functioning.

The pinhole implant should not be considered as first option in any scenario. It should be limited to challenging cases of irregular astigmatism, and it may also be considered as an adjunct treatment option to other treatment modalities (intrastromal ring segment implantation, topo guided excimer laser ablation, toric IOL implantation, among others). The implant is especially appropriate for cases with concurrent iris defect. Limitations of this approach include cases of irregular astigmatism associated with central corneal opacity, where implantation of the pinhole should not be considered. The clinical benefits of this device are limited by the amount of high order aberration. Patients with severe topographic irregularity may not achieve sufficient improvement in visual acuity with this treatment. Furthermore, after implantation, binocular indirect ophthalmoscopy is impossible to be performed. Although infrared images may be captured with an OCT or scanning laser ophthalmoscope (because of the window of infrared light transmittance of the acrylic material used to manufacture the pinhole implant), such machines offer only a two-dimensional view of the posterior segment, and the field of view is not as wide as with regular indirect ophthalmoscopy. ${ }^{11,17}$ These limitations may be addressed by the development of an infrared indirect ophthalmoscope.

Obviously, larger scale studies will investigate the visual outcomes of the suggested implant design but for the present time the scope of our study was to demonstrate the fit of this novel device in cadaver eyes, as adequate alignment is essential for not adding optical aberrations, which will fail to improve the patient's visual 
a

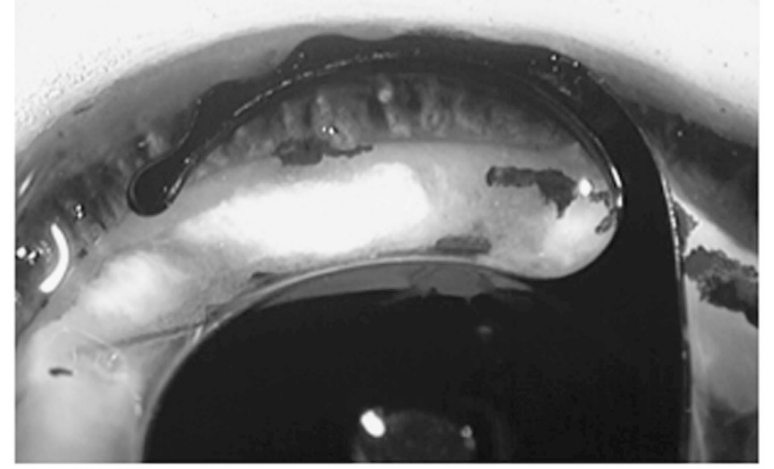

b

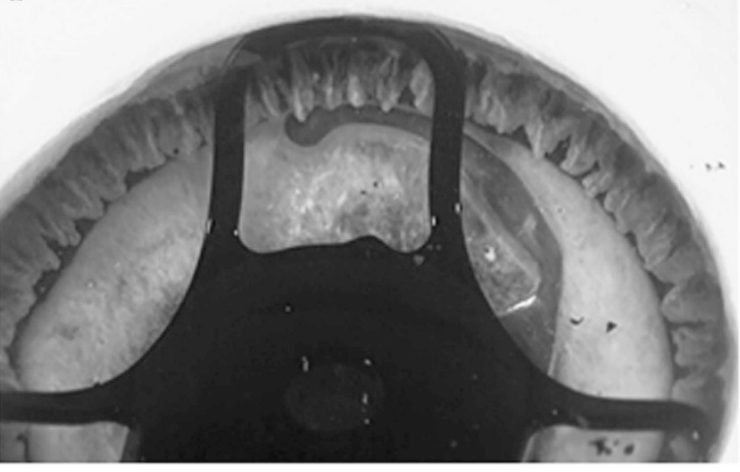

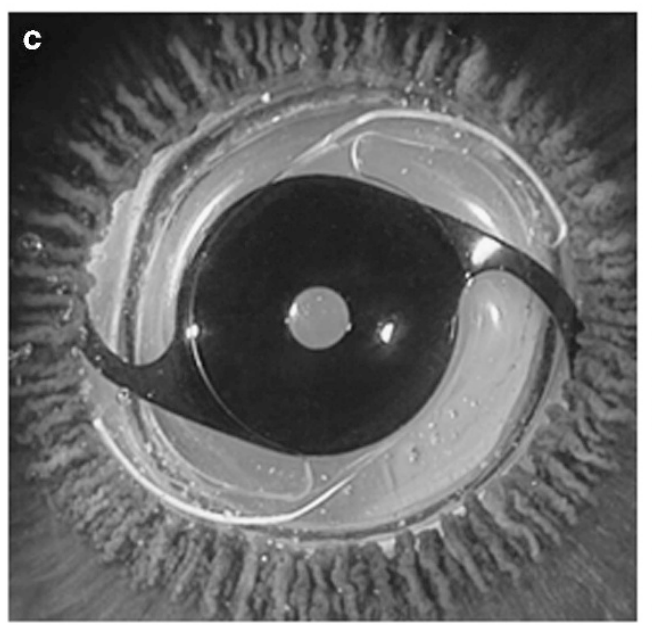
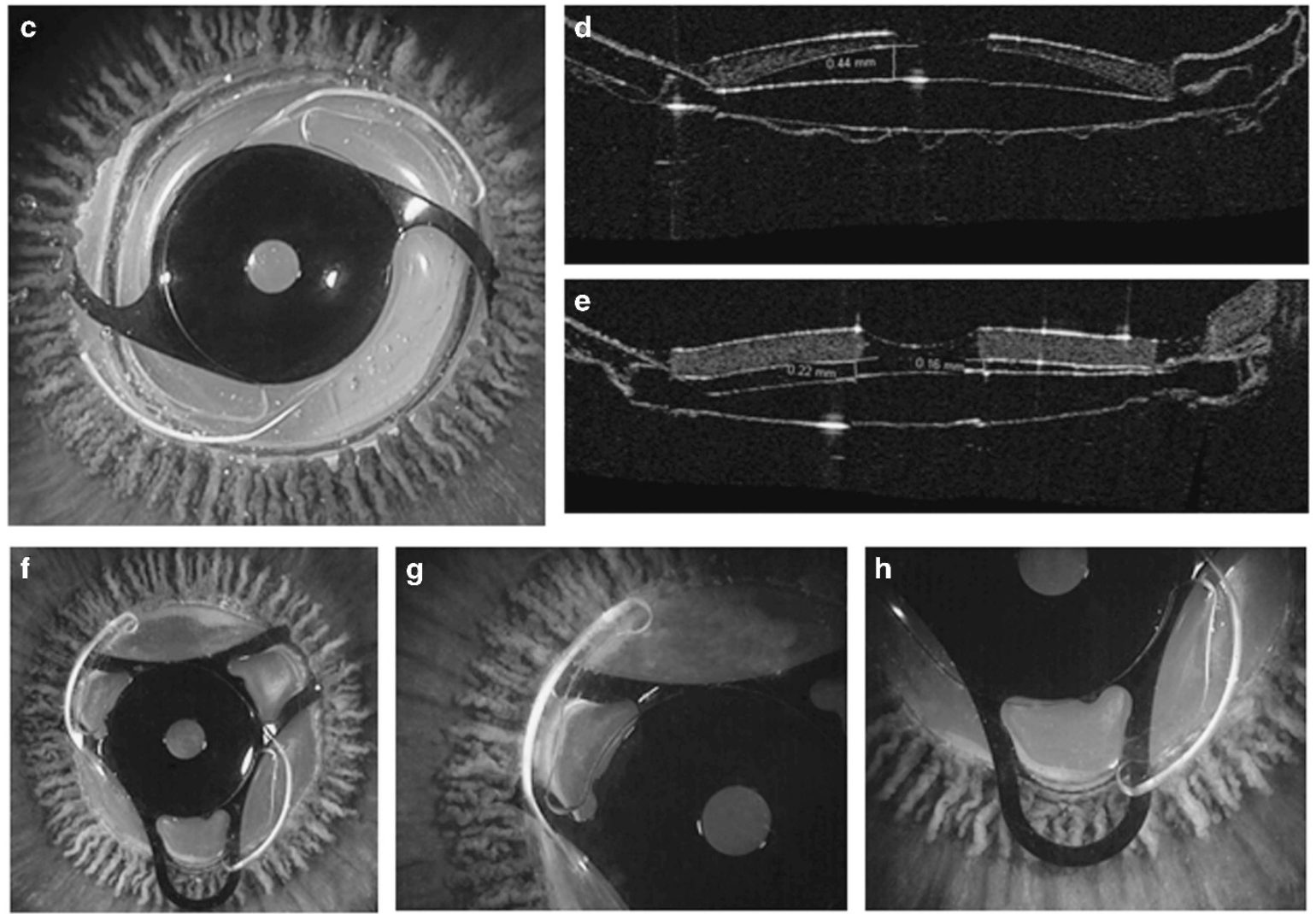

Figure 4 Post add-on implantation evaluation in cadaver eyes. Values shown in mm are ILD measurements. (a, b) Gross photographs from the anterior view after complete removal of the iris, showing the haptic components of both add-on implant models in the sulcus (a Eye \#2, after implantation of model 93L. (b) Eye \#1 after implantation of model 93E). (c, d) Imaging of the fresh eye (\#10) prepared according to the Miyake-Apple technique for experimental surgery. A single-piece hydrophobic acrylic lens was experimentally implanted inside of the capsular bag, which is completely clear. Model 93L was inserted in the sulcus and is well centered without tilt (c) Gross photograph from the Miyake-Apple view. (d) AS-OCT scan showing an interlenticular distance of $0.44 \mathrm{~mm}$ ). (e-h) Same eye (\#10) after insertion of model 93E (e) AS-OCT scan showing mild tilt of the add-on lens in relation to the in-the-bag primary IOL. (f) The add-on implant was inserted in the sulcus and is well centered. (g) Correct positioning of the haptic element in the ciliary sulcus. (h) This haptic element is posterior to the ciliary processes.

acuity. The primary outcomes of interest with the two versions of the implant in the present study were centration and tilt (angle between supplementary and primary IOLs). Centration is of crucial importance in the small-aperture lens to allow paraxial light rays to reach the fovea, while tilting is not desirable since further aberrations may be induced, ${ }^{20}$ especially in cases of polypseudophakia. ${ }^{21}$ In the present study pre-existing zonular dehiscence was identified as the main causal factor of decentration and mostly tilt. It is noteworthy that in our set up, pre-existing zonular dehiscence may be related to postmortem changes. In eyes with zonular 
weakness or dehiscence, one or more of the closed loops of the tripod version could dislocate posteriorly to the sulcus causing tilt of the add-on. If significant, this finding could induce secondary astigmatism which potentially can compromise the final clinical outcome. Localized Soemmering's ring formation has been recognized as an additional cause for piggyback IOL tilt and decentration. ${ }^{15}$ In the present study, tilt was observed in one eye in this circumstance (tripod, old model, eye \#8). A possible explanation is that the open loops of the new model were not close to the capsular bag area with prominent proliferative material and maintained their centration, while one or two of the closed loops of the old model tilted as they were on the area with a localized Soemmering's ring. In any case, a detailed preoperative examination through any available imaging modality (AS-OCT, ultrasound biomicroscopy) is highly recommended in order to detect areas of localized Soemmering's ring and position the add-on implant accordingly with loops avoiding these areas.

Another parameter of interest was the distance between the add-on implant and the primary IOL (ILD). The new and old versions of the pinhole implant have characteristics to minimize the possibility of contact between the add-on and in-the-bag IOLs, decreasing likelihood of induced refractive error and optical aberrations. Indeed, studies have found that the contact zone between piggyback IOLs varied in morphologic appearance and size, depending on the IOL material and the optic design, and this could be associated with optical effects. $^{22-24}$ Opacification of the interface (interlenticular opacification - ILO) has also been widely reported in cases where there is contact between IOLs; however, in the cases of ILO described in the literature both lenses were hydrophobic acrylic lenses fixated within the capsular bag. ${ }^{25}$ A satisfactory ILD was observed in all eyes in this study, especially for the new open-loop design. The new and old versions of the pinhole implant have design characteristics similar to the Sulcoflex lens (Rayner, West Sussex, UK), and the A4 AddOn IOL (Medicontur Medical Engineering Ltd, Zsámbék, Hungary/1stQ GmbH, Mannheim, Germany), respectively. Both are add-on lenses, which have demonstrated appropriate anatomical results in clinical studies. ${ }^{12,26}$ The older 93E version (tripod), of the pinhole implant served as a prototype only, and is no longer available. The only commercial available version is the 93L version, with two open-loop haptics, which received CE Mark in September 2016.

It is noteworthy that the ILD values reported in this study were obtained in cadaver eyes, and likely do not represent the actual ILD values that would be observed clinically with both implant versions, considering the limitations of the study model. Nevertheless, the results clearly demonstrated the relationship between zonular weakness and IOL tilt and adequate ILD between in thebag and add-on IOLs in all cases.

\section{Summary}

What was known before

- Two main factors that may cause piggyback (add-on; supplementary) IOL tilting are pre-existing asymmetrical cortical proliferation (localized Soemmering's ring) and pre-existing zonular dehiscence.

- A new add-on implant with a design based on the pinhole principle can be fixated in the ciliary sulcus to improve clinical symptoms related to irregular astigmatism, associated or not with iris defects.

What this study adds

- Both versions of the new add-on pinhole implant (openloop and tripod designs) demonstrated appropriate fixation when implanted in cadaver eyes. The risk of tilting of the add-on implant in eyes with preexisting zonular dehiscence may be higher with the tripod design.

\section{Conflict of interest}

The authors declare no conflict of interest.

\section{Acknowledgements}

Michael Anderson, COA, Moran Eye Center assisted with the AS-OCT examination. Work supported in part by an Unrestricted Grant from Research to Prevent Blindness, Inc., New York, NY, to the Department of Ophthalmology \& Visual Sciences, University of Utah. Morcher $\mathrm{GmbH}$ provided the pinhole intraocular lenses used in this study. CLCT holds a patent of the implant and has a license contract with Morcher $\mathrm{GmbH}$. The other authors have no financial or proprietary interest in any product mentioned in this paper. $\mathrm{LW}, \mathrm{MD}, \mathrm{PhD}$, reports research grants from Abbott Medical Optics, grants and personal fees from Alcon, grants from Anew Optics, grants from Advanced Visual Sciences, grants from CIMA, grants from ClarVista, grants from DORC, grants from Hoya, grants from Medicontur, grants from Perfect Lens, grants from PhysIOL, grants and personal fees from PowerVision, grants from Sharklet, grants from Shifamed, grants from Sigilon, grants from Zeiss outside the submitted work.

\section{References}

1 Fares U, Sarhan AR, Dua HS. Management of postkeratoplasty astigmatism. J Cataract Refract Surg 2012; 38(11): 2029-2039.

2 Perlman EM. An analysis and interpretation of refractive errors after penetrating keratoplasty. Ophthalmology 1981; 88: 39-45. 
3 Karabatsas CH, Cook SD, Sparrow JM. Proposed classification for topographic patterns seen after penetrating keratoplasty. Br J Ophthalmol 1999; 83(4): 403-409.

4 Schraepen P, Vandorselaer T, Trau R, Tassignon MJ. LASIK and arcuate incisions for the treatment of post-penetrating keratoplasty anisometropia and/or astigmatism. Bull Soc Belge Ophtalmol 2004; 292: 19-25.

5 Buzard K, Febbraro JL, Fundingsland BR. Laser in situ keratomileusis for the correction of residual ametropia after penetrating keratoplasty. J Cataract Refract Surg 2004; 30(5): 1006-1013.

6 Laíns I, Rosa AM, Guerra M, Tavares C, Lobo C, Silva MF, Quadrado MJ, Murta JN. Irregular astigmatism after corneal transplantation-efficacy and safety of topography-guided treatment. Cornea 2016; 35(1): 30-36.

7 Kymionis GD, Yoo SH, Ide T, Culbertson WW. Femtosecond-assisted astigmatic keratotomy for postkeratoplasty irregular astigmatism. J Cataract Refract Surg 2009; 35(1): 11-13.

8 Barnett M, Lien V, Li JY, Durbin-Johnson B, Mannis MJ. Use of Scleral Lenses and Miniscleral Lenses After Penetrating Keratoplasty. Eye Contact Lens 2016; 42(3): 185-189.

9 Dexl AK, Jell G, Strohmaier C, Seyeddain O, Riha W, Rückl T, Bachernegg A, Grabner G. Long-term outcomes after monocular corneal inlay implantation for the surgical compensation of presbyopia. J Cataract Refract Surg 2015; 41(3): 566-575.

10 Lindstrom RL, Macrae SM, Pepose JS, Hoopes PC Sr. Corneal inlays for presbyopia correction. Curr Opin Ophthalmol 2013; 24(4): 281-287.

11 Trindade CLC, Trindade BLC. Novel pinhole intraocular implant for the treatment of irregular corneal astigmatism and severe light sensitivity after penetrating keratoplasty. J Cataract Refract Surg Online Case Reports 2015; 3: 4-7.

12 Kahraman G, Amon M. New supplementary intraocular lens for refractive enhancement in pseudophakic patients. J Cataract Refract Surg 2010; 36(7): 1090-1094.

13 Falzon K, Stewart OG. Correction of undesirable pseudophakic refractive error with the Sulcoflex intraocular lens. J Refract Surg 2012; 28(9): 614-619.

14 McIntyre JS, Werner L, Fuller SR, Kavoussi SC, Hill M, Mamalis N. Assessment of a single-piece hydrophilic acrylic IOL for piggyback sulcus fixation in pseudophakic cadaver eyes. J Cataract Refract Surg 2012; 38(1): 155-162.
15 Reiter N, Werner L, Guan J, Li J, Tsaousis KT, Mamalis N, Srinivasan S. Assessment of a new hydrophilic acrylic supplementary IOL for sulcus fixation in pseudophakic cadaver eyes. Eye (Lond) 2017; 31(5): 802-809.

16 Pereira FA, Werner L, Milverton EJ, Coroneo MT. MiyakeApple posterior video analysis/photographic technique. J Cataract Refract Surg 2009; 35: 577-587.

17 Trindade CLC, Trindade FC, Trindade BC. Tiny hero against the evil axis. Video presented at the Annual Meeting of the American Society of Cataract and Refractive Surgery (ASCRS) 2017, CA, USA. Available at: http:/ /ascrs2017. conferencefilms.com/abstractawards.wcs.

18 Kim WS, Park IK, Chun YS. Quantitative analysis of functional changes caused by pinhole glasses. Invest Ophthalmol Vis Sci 2014; 55(10): 6679-6685.

19 Kanellopoulos AJ, Asimellis G, Georgiadou S. Digital pupillometry and centroid shift changes after cataract surgery. J Cataract Refract Surg 2015; 41(2): 408-414.

20 Li L, Wang K, Yan Y, Song X, Liu Z. Research on calculation of the IOL tilt and decentration based on surface fitting. Comput Math Methods Med 2013; 2013: 572530.

21 Gomaa A, Lee RM, Liu CS. Polypseudophakia for cataract surgery: 10-year follow-up on safety and stability of two poly-methyl-methacrylate (PMMA) intraocular lenses within the capsular bag. Eye (Lond) 2011; 25(8): 1090-1093.

22 Findl O, Menapace R, Rainer G, Georgopoulos M. Contact zone of piggyback acrylic intraocular lenses. J Cataract Refract Surg 1999; 25(6): 860-862.

23 Findl O, Menapace R, Georgopoulos M, Kiss B, Petternel V, Rainer G. Morphological appearance and size of contact zones of piggyback intraocular lenses. J Cataract Refract Surg 2001; 27(2): 219-223.

24 Hesse RJ. Refractive changes produced by capsule contraction after piggyback acrylic intraocular lens implantation. J Cataract Refract Surg 2002; 28(12): 2229-2230.

25 Werner L, Apple DJ, Pandey SK, Solomon KD, Snyder ME, Brint SF, Gayton JL, Shugar JK, Trivedi RH, Izak AM. Analysis of elements of interlenticular opacification. Am J Ophthalmol 2002; 133(3): 320-326.

26 Scharioth GB. New add-on intraocular lens for patients with age-related macular degeneration. J Cataract Refract Surg 2015; 41(8): 1559-1563. 